\title{
Overexpression of Wilms' tumor 1 in skin lesions of psoriasis is associated with abnormal proliferation and apoptosis of keratinocytes
}

\author{
RUIFANG WU ${ }^{1}$, YUAN LIAO $^{1}$, WEIYUN SHEN ${ }^{2}$, YU LIU $^{1}$, JIANZHONG ZHANG $^{3}$, \\ MIN ZHENG $^{4}$, GENGHUI CHEN $^{5}$, YUWEN SU ${ }^{1}$, MING ZHAO $^{1}$ and QIANJIN LU ${ }^{1}$ \\ ${ }^{1}$ Department of Dermatology, Hunan Key Laboratory of Medical Epigenomics; \\ ${ }^{2}$ Department of Anesthesiology, The Second Xiangya Hospital of Central South University, Changsha, Hunan 410011; \\ ${ }^{3}$ Department of Dermatology, Peking University People's Hospital, Beijing 100044; \\ ${ }^{4}$ Department of Dermatology, The Second Affiliated Hospital, Zhejiang University School of Medicine, \\ Hangzhou, Zhejiang 310009; ${ }^{5}$ Beijing Wenfeng Tianji Pharmaceuticals Ltd., Beijing 100027, P.R. China
}

Received February 9, 2018; Accepted July 12, 2018

DOI: $10.3892 / \mathrm{mmr} .2018 .9391$

\begin{abstract}
Psoriasis vulgaris (PV) is a chronic inflammatory skin disease, which is characterized by the abnormal proliferation and apoptosis of keratinocytes. Previous studies have demonstrated that transcription factor Wilms' tumor 1 (WT1) is involved in a number of pathophysiological processes, including organ development, tumorigenesis and cell proliferation. However, the role of WT1 in PV remains unclear. In the present study, WT1 expression was analyzed by reverse transcription-quantitative polymerase chain reaction and western blot analyses. WT1 was stably overexpressed or inhibited in HaCaT cells using Lipofectamine $^{\circledR} 2000$, and cell proliferation and apoptosis were determined using a Cell Counting Kit-8 or Fluorescein Isothiocyanate Annexin V Apoptosis Detection kit II, respectively. We demonstrated that compared with normal controls, the mRNA and protein expression levels of WT1 were significantly increased in non-lesional skins (human, $\mathrm{P}<0.0001$ and $\mathrm{P}=0.0291$, respectively; mouse, $\mathrm{P}=0.0020$ and $\mathrm{P}=0.0150$, respectively) and lesional skins (human, $\mathrm{P}<0.0001$ and $\mathrm{P}=0.0060$, respectively; mouse, $\mathrm{P}=0.0010$ and $\mathrm{P}=0.0172$, respectively) of patients with $\mathrm{PV}$, in addition to the imiquimod (IMQ)-induced psoriasis-like mouse model. WT1 mRNA and protein expression levels in lesional skins were slightly increased compared with those in non-lesional skins from patients with psoriasis $(\mathrm{P}=0.2510$ and $\mathrm{P}=0.1690$, respectively) and IMQ-treated mice $(\mathrm{P}=0.9590$
\end{abstract}

Correspondence to: Professor Qianjin Lu or Professor Ming Zhao, Department of Dermatology, Hunan Key Laboratory of Medical Epigenomics, The Second Xiangya Hospital of Central South University, 139 Renmin Middle Road, Changsha, Hunan 410011, P.R. China

E-mail: qianlu5860@csu.edu.cn

E-mail: zhaoming307@csu.edu.cn

Key words: psoriasis vulgaris, Wilms' tumor 1, keratinocytes, apoptosis, proliferation and $\mathrm{P}=0.2552$, respectively), although there were no statistical differences. Knockdown of WT1 inhibited the proliferation of HaCaT cells [day (D)4, P=0.0454; D5, P=0.0021] and promoted their apoptosis $(\mathrm{P}=0.0007)$, while overexpressing WT1 exhibited the opposite effects (proliferation D3, $\mathrm{P}=0.0216$; D4, $\mathrm{P}=0.0356$; D5, $\mathrm{P}=0.0188$; apoptosis, $\mathrm{P}=0.0003$ ). Furthermore, it was identified that the inflammatory cytokines interleukin-17A (IL-17A), interferon- $\gamma$ and IL-22 induced the overexpression of WT1 in HaCaT cells. The results of the present study suggested that inflammatory cytokine-induced WT1 overexpression may promote the formation of psoriatic skin lesions via regulation of the proliferation and apoptosis of keratinocytes.

\section{Introduction}

Psoriasis is a chronic inflammatory skin disease, which may severely impact the quality of life of patients and manifests as erythematous plaques covered with silvery-white scales (1). Psoriatic lesions are characterized by epidermal hyperplasia with parakeratosis, loss of the granular layer, acanthosis, aberrant differentiation, proliferation of keratinocytes and marked infiltration of immune cells into the dermis or epidermis (2). The pathogenesis of psoriasis is complex, and the exact underlying mechanism of the factors involved remains elusive. The abnormal proliferation of keratinocytes is a key feature of psoriasis, which results in epidermal hyperplasia and the morphological characteristics of psoriasis (3). It is widely accepted that the abnormal growth dynamics of keratinocytes are due to the dysregulation of cytokines and growth factors, which are secreted by infiltrated immune cells in the skin lesions $(4,5)$. Among these, interferon- $\gamma(\mathrm{IFN}-\gamma)$, interleukin (IL)-17A and IL-22 have been demonstrated to be increased and to serve important roles in the development of the skin lesions observed in patients with psoriasis (6-10). However, the molecular mechanisms involved in this process remain unclear.

The Wilms' tumor 1 (WT1) gene, which maps to chromosome 11 p13 and contains 10 exons, encodes a DNA-binding transcription factor that is involved in the regulation of 
human cell growth and differentiation (11). This gene locus is frequently mutated in patients with Wilms' tumor. In addition, alterations in this gene have been identified in a variety of cancer types, including breast cancer, renal cell cancer, ovarian cancer, lung cancer, melanoma and acute leukemia (12-18). In these types of cancer, WT1 acts as either an oncogene or a tumor suppressor gene, depending on the different cellular characteristics (19-21). However, to the best of our knowledge, there have been no previous studies focusing on the expression and role of WT1 in the formation of psoriatic skin lesions.

The present study detected the expression of WT1 in the non-lesional skins and skin lesions from patients with psoriasis vulgaris (PV) and an imiquimod (IMQ)-induced psoriasis-like mouse model. The effect of WT1 on the proliferation and apoptosis of keratinocytes was subsequently investigated. It was revealed that WT1 expression was significantly increased in non-lesional skin tissues and psoriatic skin lesions. Overexpressing WT1 promoted keratinocyte proliferation and inhibited apoptosis. In addition, certain inflammatory cytokines upregulated WT1 in keratinocytes. These findings indicated that WT1 may serve an important role in the formation of skin lesions associated with PV.

\section{Materials and methods}

Human subjects. A total of 20 psoriatic patients who were diagnosed with PV by pathological examination were recruited from outpatient clinics at the Second Xiangya Hospital of Central South University (Changsha, China). Psoriasis disease activity was assessed using psoriasis area and severity index (PASI) scores (22), and blood samples and lesional skins were collected. Non-lesional skin tissues were obtained from 10 of the patients simultaneously. Patient information is presented in Table I. Blood samples were collected from 20 sex- and age-matched healthy controls who were recruited from the medical staff at the Second Xiangya Hospital. Normal skin tissues were obtained from the outpatient operating room at the Department of Dermatology at the Second Xiangya Hospital. The information of all healthy controls is presented in Table II. The present study was approved by the Ethics Committee of the Second Xiangya Hospital of Central South University. Written informed consent was obtained from all subjects.

Assessment of PASI scores. For determining the severity and extent of psoriasis, PASI scoring was used $(22,23)$. In the four regions of the body, namely the head (h), upper extremities $(\mathrm{u})$, lower extremities (1) and torso (t), the characteristics of the disease, including erythema (E), infiltration (I) and desquamation (D), were evaluated with a score of 1-4, and the involved area (A) of psoriatic lesions was evaluated with a score of 1-6 (Table III). PASI total scores ranges between 0 and 72. Higher scores indicate greater psoriasis severity. The formula used to calculate the total PASI score is as follows: PASI $=\left(E_{h}+I_{h}+D_{h}\right) \times A_{h} \times 0.1+\left(E_{u}+I_{u}+D_{u}\right)$ $\mathrm{xA}_{\mathrm{u}} \mathrm{x} 0.2+\left(\mathrm{E}_{\mathrm{t}}+\mathrm{I}_{\mathrm{t}}+\mathrm{D}_{\mathrm{t}}\right) \times \mathrm{A}_{\mathrm{t}} \mathrm{x} 0.3+\left(\mathrm{E}_{\mathrm{l}}+\mathrm{I}_{\mathrm{l}}+\mathrm{D}_{\mathrm{l}}\right) \mathrm{xA}_{\mathrm{l}} \mathrm{x} 0.4$

IMQ-induced psoriasis-like mouse model. Female BALB/c mice (age, 6-8 weeks; 19.0-20.5 g) were purchased from Shanghai SLAC Laboratory Animal Co., Ltd. (Shanghai, China). All mice were maintained in specific pathogen-free conditions $\left(20-24^{\circ} \mathrm{C}\right.$; relative humidity, 50-55\%; $12 \mathrm{~h}$ light/dark cycle) with free access to food and water. The IMQ-induced psoriasis-like mouse model was established as previously described (24). The mice were treated with a daily topical dose of 62.5 mg 5\% IMQ cream (cat. no. H20030128; Sichuan Med-Shine Pharmaceutical Co., Ltd., Chengdu, China) on their shaved backs for 7 consecutive days. The control mice were treated with the same dose of vehicle cream. All procedures were approved and supervised by the Animal Care and Use Committee of the Second Xiangya Medical School of Central South University.

Cell isolation and culture. Peripheral blood mononuclear cells (PBMCs) were separated from the peripheral blood of healthy controls and patients with psoriasis by density gradient centrifugation at $18^{\circ} \mathrm{C}$ and $600 \mathrm{x}$ g for $30 \mathrm{~min}$ (GE Healthcare, Chicago, IL, USA). The cells were cultured in RPMI 1640 medium (Gibco; Thermo Fisher Scientific, Inc., Waltham, MA, USA) supplemented with $10 \%$ fetal bovine serum (FBS; HyClone; GE Healthcare Life Sciences, Logan, UT, USA) at $37^{\circ} \mathrm{C}$ in $5 \% \mathrm{CO}_{2}$, or collected directly for subsequent experiments. HaCaT cells (cat no. BNCC101683; BeNa Culture Collection, Beijing, China), which were stored in liquid nitrogen, were revived and cultured in Dulbecco's modified Eagle's medium (DMEM; Gibco; Thermo Fisher Scientific, Inc.) supplemented with $10 \% \mathrm{FBS}$ at $37^{\circ} \mathrm{C}$ in $5 \% \mathrm{CO}_{2}$. The medium was refreshed every 2 days and the cells were subcultured when $90 \%$ confluence was reached.

Reverse transcription-quantitative polymerase chain reaction (RT-qPCR). Total RNA was extracted from the cells or skin tissues using TRIzol ${ }^{\circledR}$ reagent (Invitrogen; Thermo Fisher Scientific, Inc.), and a NanoDrop spectrophotometer (ND-2000; Thermo Fisher Scientific, Inc.) was used for RNA quality control. The mRNA was reverse-transcribed using a PrimeScript ${ }^{\circledR}$ RT reagent kit with gDNA Eraser (Takara Biotechnology Co., Ltd., Dalian, China). Each test used $1 \mu \mathrm{g}$ total RNA and was performed according to the manufacturer's protocol. qPCR was subsequently performed using the SYBR Premix Ex Taq II (Tli RnaseH Plus; Takara Biotechnology Co., Ltd.) using a LightCycler ${ }^{\circledR} 96$ (Roche Diagnostics, Basel, Switzerland) thermocycler. The thermocycling conditions were as follows: Initial denaturation at $95^{\circ} \mathrm{C}$ for $30 \mathrm{sec}$, followed by 45 cycles of $95^{\circ} \mathrm{C}$ for $5 \mathrm{sec}$ and $60^{\circ} \mathrm{C}$ for $20 \mathrm{sec}$, and a final extension $\left(95^{\circ} \mathrm{C}\right.$ for $1 \mathrm{sec}, 65^{\circ} \mathrm{C}$ for $15 \mathrm{sec}$ and $95^{\circ} \mathrm{C}$ for $\left.1 \mathrm{sec}\right)$. The relative expression of the target genes was calculated using the $2^{-\Delta \Delta \mathrm{Cq}}$ method (25) and normalized against the GAPDH internal control. Detailed information on the primers used is summarized in Table IV.

Western blot analysis. The cells or skin tissues were lysed in radioimmunoprecipitation assay buffer supplemented with protease and phosphatase inhibitors (Beyotime Institute of Biotechnology, Haimen, China). The proteins were quantified using a Bradford assay (Pierce; Thermo Fisher Scientific, Inc.) and $100 \mu \mathrm{g}$ protein from each sample was loaded for $8 \%$ SDS-PAGE. Following the transfer of proteins onto a polyvinylidene fluoride membrane, the membrane was blocked with $5 \%$ skim milk in PBS with $0.1 \%$ Tween-20 at room temperature for 1 h. Rabbit anti-WT1 (1:1,000; cat no. ab89901; 
Table I. Information on patients with psoriasis vulgaris.

\begin{tabular}{lcr}
\hline Sample ID & Age/sex & PASI score \\
\hline 1 & $36 / \mathrm{F}$ & 0.7 \\
2 & $25 / \mathrm{F}$ & 9.5 \\
3 & $24 / \mathrm{F}$ & 8.6 \\
4 & $54 / \mathrm{M}$ & 10.6 \\
5 & $29 / \mathrm{M}$ & 2.4 \\
6 & $45 / \mathrm{M}$ & 5.9 \\
7 & $46 / \mathrm{M}$ & 10.2 \\
8 & $18 / \mathrm{F}$ & 2.4 \\
9 & $30 / \mathrm{M}$ & 0.2 \\
10 & $45 / \mathrm{M}$ & 10.7 \\
11 & $38 / \mathrm{F}$ & 8.1 \\
12 & $22 / \mathrm{M}$ & 18.1 \\
13 & $45 / \mathrm{M}$ & 8.0 \\
14 & $30 / \mathrm{M}$ & 4.0 \\
15 & $42 / \mathrm{F}$ & 4.5 \\
16 & $48 / \mathrm{M}$ & 8.8 \\
17 & $27 / \mathrm{M}$ & 8.9 \\
18 & $30 / \mathrm{M}$ & 12.5 \\
19 & $40 / \mathrm{M}$ & 11.8 \\
20 & $20 / \mathrm{M}$ & 10.4 \\
\hline
\end{tabular}

F, female; M, male; PASI, psoriasis area and severity index.

Abcam, Cambridge, UK) and goat anti-GAPDH (1:2,000; cat no. ab9483; Abcam) primary antibodies were incubated with the membrane at $4^{\circ} \mathrm{C}$ overnight. Horseradish peroxidase (HRP) goat anti-rabbit immunoglobulin $(\mathrm{Ig}) \mathrm{G}(\mathrm{H}+\mathrm{L})$ (1:5,000; cat no. AS014; ABclonal Biotech Co., Ltd., Woburn, MA, USA) and HRP donkey anti-goat $\operatorname{IgG}(\mathrm{H}+\mathrm{L})(1: 5,000$; cat no. A00178; GenScript, Piscataway, NJ, USA) secondary antibodies were incubated at room temperature for $2 \mathrm{~h}$. The data was analyzed using a GE-ImageQuant LAS 4000 mini (GE Healthcare). The quantification of WT1 was normalized against GAPDH by densitometric analysis with Image-Pro Plus 6.0 (Media Cybernetics, Inc., Rockville, MD, USA). The images were cropped for presentation.

Immunohistochemistry. Skin tissues were fixed in formalin at room temperature overnight and embedded in paraffin. The $6-\mu \mathrm{m}$-thick sections were stained with hematoxylin for $10 \mathrm{~min}$, and then stained with eosin for $2 \mathrm{~min}$ at room temperature. For immunohistochemistry, the sections were stained with rabbit anti-WT1 (1:200; cat no. ab89901; Abcam) or rabbit anti-Ki67 polyclonal antibodies (1:100; cat no. ab15580; Abcam) at $4^{\circ} \mathrm{C}$ overnight, according to the manufacturer's protocol. Image analysis was performed using a DMI 4000B microscope (magnification, x100) and Leica Qwin Std analysis software version 3 (both Leica Microsystems GmbH, Wetzlar, Germany).

Small interfering (si)RNA and plasmid transfection of HaCaT cells. A total of $12 \mathrm{~h}$ prior to transfection, the HaCaT cells were seeded in a 6 -well cell culture plate at $6 \times 10^{3}$ cells/well. The cells were transfected with $20 \mathrm{nM}$ WT1 siRNA, $5 \mu \mathrm{g}$ WT1 plasmid
Table II. Information on healthy controls.

\begin{tabular}{|c|c|}
\hline Sample ID & Age/sex \\
\hline 1 & 16/Male \\
\hline 2 & 31/Female \\
\hline 3 & 35/Female \\
\hline 4 & 24/Female \\
\hline 5 & 19/Female \\
\hline 6 & 25/Male \\
\hline 7 & 32/Male \\
\hline 8 & 28/Male \\
\hline 9 & 50/Female \\
\hline 10 & 55/Female \\
\hline 11 & 26/Female \\
\hline 12 & 35/Male \\
\hline 13 & 34/Female \\
\hline 14 & 54/Female \\
\hline 15 & 39/Female \\
\hline 16 & 25/Male \\
\hline 17 & 37/Female \\
\hline 18 & 29/Male \\
\hline 19 & 48/Female \\
\hline 20 & 35/Female \\
\hline
\end{tabular}

B, Blood samples

\begin{tabular}{rl}
\hline Sample ID & Age/sex \\
\hline 1 & $39 /$ Male \\
2 & $45 /$ Male \\
3 & $37 /$ Male \\
4 & $26 /$ Male \\
5 & $22 /$ Female \\
6 & $38 /$ Female \\
7 & $42 /$ Male \\
8 & $47 /$ Male \\
9 & $29 /$ Male \\
10 & $25 /$ Female \\
11 & $38 /$ Male \\
12 & $32 /$ Male \\
13 & $35 /$ Male \\
14 & $29 /$ Female \\
15 & $43 /$ Male \\
16 & $36 /$ Male \\
17 & $27 /$ Female \\
18 & $35 /$ Male \\
19 & $44 /$ Male \\
20 & $31 /$ Male \\
&
\end{tabular}

or their corresponding controls using Lipofectamine ${ }^{\circledR} 2000$ (Invitrogen, Thermo Fisher Scientific, Inc., USA) for $6 \mathrm{~h}$ in Opti-minimum essential medium (Opti-MEM; Gibco; 
Table III. Psoriasis area and severity index evaluation criteria.

\begin{tabular}{|c|c|c|c|c|c|c|c|}
\hline \multirow[b]{2}{*}{ Factor } & \multicolumn{7}{|c|}{ Grade } \\
\hline & 0 & 1 & 2 & 3 & 4 & 5 & 6 \\
\hline $\begin{array}{l}\text { Erythema (E) } \\
\text { Infiltration (I) } \\
\text { Desquamation (D) }\end{array}$ & None & Mild & Medium & Severe & Very severe & - & - \\
\hline $\begin{array}{l}\text { Involved area of the } \\
\text { psoriatic lesions (A) \% }\end{array}$ & 0 & $<10$ & $10-29$ & $30-49$ & $50-69$ & $70-89$ & $90-100$ \\
\hline
\end{tabular}

Table IV. Primer sequences, product sizes and annealing temperatures.

\begin{tabular}{llcc}
\hline Gene name & \multicolumn{1}{c}{ Primer sequence } & Annealing temperature, ${ }^{\circ} \mathrm{C}$ & Product size, bp \\
\hline Human GAPDH-F & 5'-ATGGGGAAGGTGAAGGTCG-3' & 60 & 108 \\
Human GAPDH-R & 5'-GGGGTCATTGATGGCAACAATA-3' & 60 & 147 \\
Human WT1-F & 5'-TTGAATGCATGACCTGGAAT-3' & & 123 \\
Human WT1-R & 5'-CCTGAATGCCTCTGAAGACA-3' & 60 & 128 \\
Mouse GAPDH-F & 5'-AGGTCGGTGTGAACGGATTTG-3' & 60 & \\
Mouse GAPDH-R & 5'-TGTAGACCATGTAGTTGAGGTCA-3' & & \\
Mouse WT1-F & 5'-GAGAGCCAGCCTACCATCC-3' & & \\
Mouse WT1-R & 5'-GGGTCCTCGTGTTTGAAGGAA-3' & & \\
\hline
\end{tabular}

F, forward; R, reverse; WT1, Wilms' tumor 1.

Thermo Fisher Scientific, Inc.). The Opti-MEM was removed and replaced with DMEM supplemented with $10 \%$ FBS for cell culture. The WT1 expression plasmid and the empty control plasmid were purchased from Vigene Biosciences, Inc. (Rockville, MD, USA). The WT1 and negative control siRNAs (sequences unavailable) were purchased from Invitrogen (Thermo Fisher Scientific, Inc.). The WT1 siRNA consisted of a mixture of two oligos: 5'-AAATATCTCTTATTGCAGCCT GGGT-3' and 5'-TTTCACACCTGTATGTCTCCTTTGG-3'.

Cell Counting Kit (CCK)-8 assay. HaCaT cells were seeded in 96-well plates in triplicate and transfected with WT1 siRNA or the WT1 overexpression plasmid as described above. The cells were cultured in $100 \mu \mathrm{l}$ DMEM with $10 \% \mathrm{FBS}$ for $24,48,72,96$ or $120 \mathrm{~h}$. The CCK-8 kit (Beyotime Institute of Biotechnology) was used to evaluate cell proliferation. A total of $10 \mu$ CCK- 8 solution was added to each well and the cells were incubated for $3 \mathrm{~h}$ at $37^{\circ} \mathrm{C}$ in $5 \% \mathrm{CO}_{2}$. The cell viability was detected at $450 \mathrm{~nm}$ using an EnSpire Multimode Plate Reader (PerkinElmer, Inc., Waltham, MA, USA).

Cellular apoptosis assay. HaCaT cells were seeded in 24-well plates in triplicate and transfected with WT1 siRNA or the WT1 overexpression plasmid as described above. After 24 or $48 \mathrm{~h}$, the level of cellular apoptosis was detected using a Fluorescein Isothiocyanate Annexin V Apoptosis Detection kit II (BD Pharmingen; BD Biosciences, Franklin Lakes, NJ, USA), according to the manufacturer's protocol. The data were acquired using a flow cytometer (BD Canto II; BD Biosciences) and analyzed using FlowJo software version 10 (FlowJo LLC, Ashland, OR, USA).

Cytokine stimulation of HaCaT cells. HaCaT cells were stimulated with $0,10,20$ or $50 \mathrm{ng} / \mathrm{ml}$ IL-17A, IFN- $\gamma$ or IL-22 (all PeproTech, Inc., Rocky Hill, NJ, USA). The cells were collected after $24 \mathrm{~h}$ for RT-qPCR analysis.

Statistical analysis. Data are presented as the mean \pm standard error of the mean of at least three experiments. Statistical analysis was performed using GraphPad Prism 6.0 software (GraphPad Software, Inc., La Jolla, CA, USA) and $\mathrm{P}<0.05$ was considered to indicate a statistically significant difference. The data were assessed for normality of distribution and a similar variance between groups. A two-tailed unpaired Student's t-test was used for comparisons between two groups and one-way analysis of variance with the corresponding post-hoc test (Bonferroni or Dunnett's) were used for the comparison of multiple groups. When the data were not normally distributed or there were not equal variances between two groups, a two-tailed Mann-Whitney U-test was used for statistical analysis. Correlation analysis was performed using a Spearman's $r$ test.

\section{Results}

WT1 expression is elevated in non-lesional and lesional skins of patients with psoriasis and IMQ-induced psoriasis-like model mice. To examine the role of WT1 in the pathogenesis of psoriasis, the mRNA expression level of WT1 was initially 


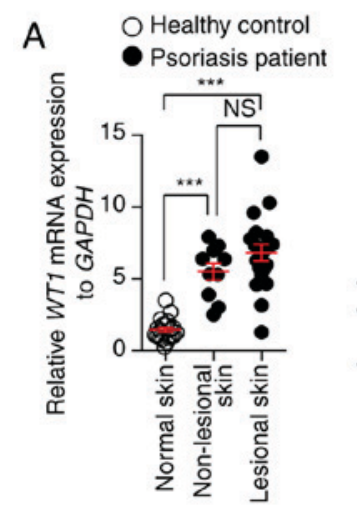

D

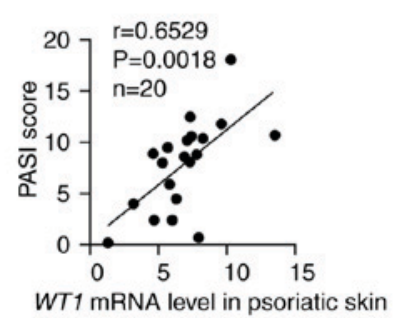

B WT1

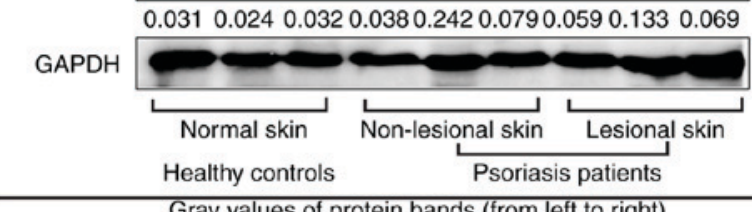

Gray values of protein bands (from left to right) GAPDH $4560.3 \quad 2950.13138 .73048 .83671 .6 \quad 3283.13447 .14449 .45451 .9$

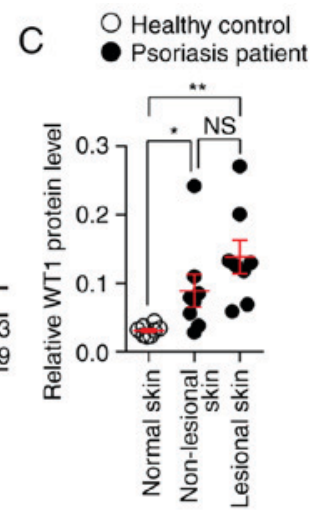

E

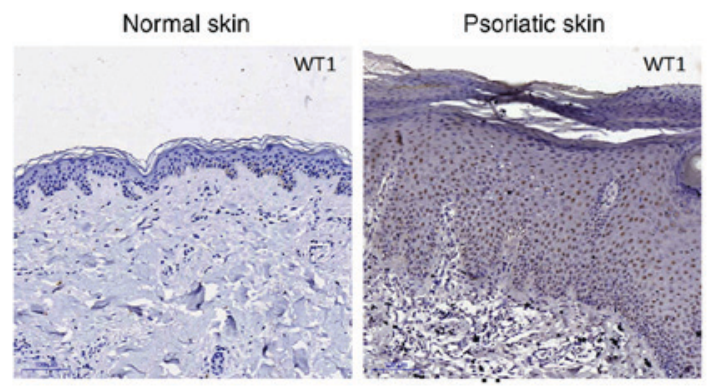

F

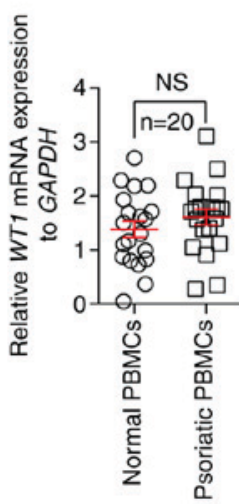

G



Gray values of protein bands (from left to right) WT1 $\quad 130.23216 .65139 .07216 .23233 .9866 .124$ GAPDH $1479.9 \quad 1504.51806 .1 \quad 1832.5 \quad 2228.3 \quad 1502.8$
$\mathrm{H}$

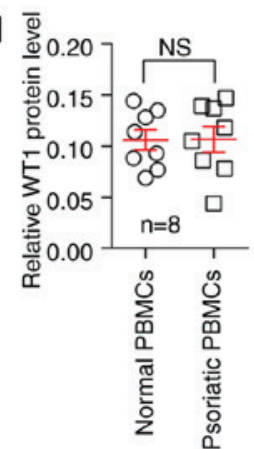

Figure 1. WT1 expression is elevated in non-lesional and lesional skins from patients with psoriasis. (A) The mRNA expression of WT1 in non-lesional (n=10) and lesional skins $(\mathrm{n}=20)$ from patients with psoriasis and normal skin samples $(\mathrm{n}=20)$ from healthy controls (non-lesional skin vs. normal skin, $\mathrm{P}<0.0001$; lesional skin vs. normal skin, $\mathrm{P}<0.0001$; non-lesional skin vs. lesional skin, $\mathrm{P}=0.2510)$. The protein expression of WT1 in non-lesional and lesional skins from psoriasis patients $(n=8)$ and normal skin samples from healthy controls $(n=8)$ was assessed. (B) A representative image of the western blotting and (C) statistical analysis of the data for WT1 protein expression (non-lesional skin vs. normal skin, $\mathrm{P}=0.0291$; lesional skin vs. normal skin, $\mathrm{P}=0.0060$; non-lesional skin vs. lesional skin, $\mathrm{P}=0.1690$ ). (D) Correlation between WT1 mRNA expression in psoriatic skin lesions and the PASI scores of patients with psoriasis ( $\mathrm{r}=0.6529$; $\mathrm{P}=0.0018 ; \mathrm{n}=20)$. (E) Immunostaining of WT1 in skin lesions from psoriasis patients and normal skin samples from healthy controls ( $=6$; magnification, $\mathrm{x} 100)$. (F) The mRNA expression levels of WT1 in PBMCs from patients with psoriasis and normal controls ( $\mathrm{n}=20$; $\mathrm{P}=0.2982$ ). The protein expression of WT1 in PBMCs from patients with psoriasis and normal controls $(n=8)$. $(\mathrm{G})$ A representative image of the western blotting and $(\mathrm{H})$ statistical analysis of the data $(\mathrm{P}=0.9634)$ for WT1 protein expression. Data are pooled from two independent experiments (A, D and F) or are representative of three independent experiments (B, C, E, G and H). Data are presented as the mean \pm standard error of the mean. ${ }^{*} \mathrm{P}<0.05,{ }^{* * *} \mathrm{P}<0.01,{ }^{* * * *} \mathrm{P}<0.001$. NS, not significant. One-way analysis of variance with Bonferroni post hoc test (A and C), Spearman's $r$ test (D) or two-tailed unpaired Student's t-test (F and H) were used for analysis. PASI, psoriasis area and severity index; PBMC, peripheral blood mononuclear cell; WT1, Wilms' tumor 1.

determined in 10 non-lesional skins and 20 lesional skins taken from patients with psoriasis and skin samples taken from 20 normal human controls using RT-qPCR. The results demonstrated that the mRNA expression of WT1 was significantly increased in non-lesional skins and lesional skins from patients with PV, compared with the normal controls (Fig. 1A). Similarly, the protein expression of WT1 in non-lesional skins and lesional skins from patients with PV was also increased compared with the normal controls (Fig. 1B and C). The results suggested that WT1 mRNA and protein expression levels in lesional skins were slightly increased compared with those in non-lesional skins from patients with psoriasis, although there were no statistical differences (Fig. 1A-C). Notably, the WT1 mRNA expression level in the psoriatic skin lesions was positively correlated with the PASI scores of the patients (Fig. 1D). In addition, immunohistochemistry with anti-WT1 antibodies was used to detect the expression and location of WT1 in the skin tissues. It was confirmed that the expression of WT1 was primarily enhanced in the epidermis of the psoriatic skin lesions (Fig. 1E). However, no significant differences were observed in the mRNA and protein expression levels of WT1 in the PBMCs of patients with PV compared with the normal controls (Fig. 1F-H).

Furthermore, the present study detected the WT1 expression in an IMQ-induced psoriasis-like mouse model, which closely 
A

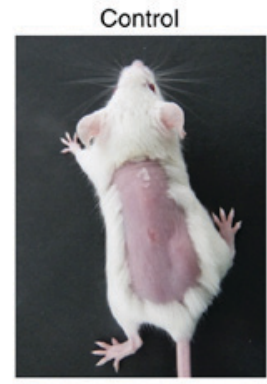

C

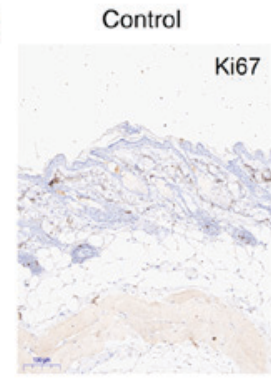

IMQ-treated


B



D



IMQ-treated

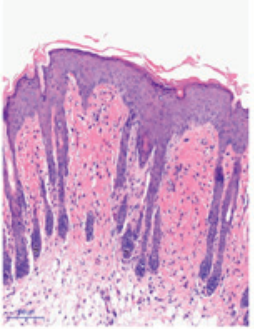



F WT1

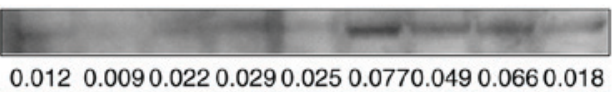

G

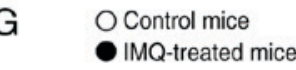

GAPDH

0.0120 .0090 .0220 .0290 .0250 .0770 .0490 .0660 .018
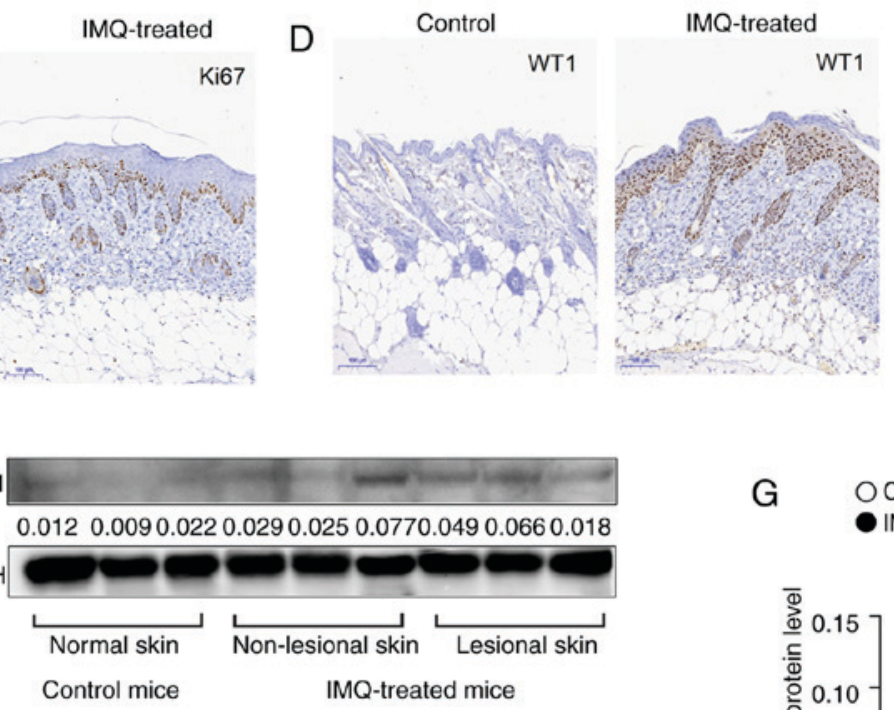

Gray values of protein bands (from left to right)


GAPDH $6205.93589 .4 \quad 3839.33893 .4 \quad 3525.6 \quad 3816 \quad 4113.4 \quad 3449.24966 .5$

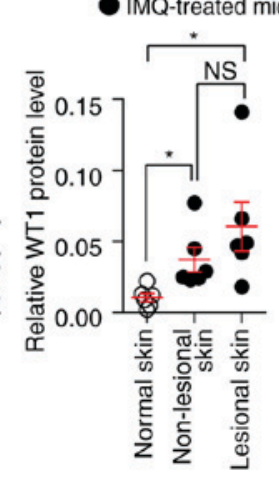

Figure 2. WT1 expression is elevated in non-lesional and lesional skins of the IMQ-induced psoriasis-like mouse model. IMQ cream was painted on the shaved backs of BALB/c mice for 7 consecutive days. (A) Phenotypic presentation and (B) hematoxylin and eosin staining of the skin lesions from IMQ-treated mice. Immunostaining of (C) Ki67 or (D) WT1 in skin lesions derived from IMQ-treated mice and normal skin samples derived from control mice. Magnifications, $\mathrm{x} 100$. (E) The mRNA expression of WT1 in non-lesional $(\mathrm{n}=12)$ and lesional skins $(\mathrm{n}=12)$ from IMQ-treated mice and normal skin samples $(\mathrm{n}=12)$ derived from control mice (non-lesional skin vs. normal skin, $\mathrm{P}=0.0020$; lesional skin vs. normal skin, $\mathrm{P}=0.0010$; non-lesional skin vs. lesional skin, $\mathrm{P}=0.9590$ ). (G) The protein expression of WT1 in non-lesional and lesional skins from IMQ-treated mice $(\mathrm{n}=6)$ and normal skin samples derived from control mice $(n=6)$ was assessed. Representative image of the $(F)$ western blotting and $(G)$ statistical analysis of the data for WT1 protein expression (non-lesional skin vs. normal skin, $\mathrm{P}=0.0150$; lesional skin vs. normal skin, $\mathrm{P}=0.0172$; non-lesional skin vs. lesional skin, $\mathrm{P}=0.2552$ ). Data (A-D and $\mathrm{F}$ ) are representative of at least three independent experiments with three to six samples per group. Data $(E$ and $G)$ are pooled from two independent experiments. Data are presented as the mean \pm standard error of the mean. ${ }^{*} \mathrm{P}<0.05,{ }^{* *} \mathrm{P}<0.01$. NS, not significant. One-way analysis of variance with Bonferroni post hoc test (E and G) was used. IMQ, imiquimod; WT1, Wilms' tumor 1.

resembles the human psoriasis phenotype, according to a previously published study (24). IMQ cream was applied to the shaved backs of BALB/c mice for 7 consecutive days to establish this model. As expected, the IMQ-treated mice developed typical psoriasis-like lesions with evident clinical and histological alterations (Fig. 2A and B). The results demonstrated that the expression of Ki67, a marker exclusively associated with cell proliferation, was significantly increased in the IMQ-treated mice compared with the controls, which indicated that the excessive proliferation of keratinocytes was induced by the IMQ (Fig. 2C). Consistent with the results of the human samples, the mice exposed to IMQ expressed significantly higher levels of WT1 mRNA and protein in their non-lesional skins and skin lesions compared with the vehicle-exposed mice (Fig. 2D-G).
Overexpressing WT1 in keratinocytes promotes proliferation and inhibits apoptosis. Abnormal proliferation and apoptosis of keratinocytes is a pathological hallmark of psoriasis (26). To examine the role of increased WT1 in the pathogenesis of $\mathrm{PV}, \mathrm{HaCaT}$ cells were transfected with a WT1 overexpression plasmid or a control plasmid. The results of the western blot analysis demonstrated that the WT1 protein was successfully overexpressed in the WT1 expression plasmid-transfected cells (Fig. 3A). A CCK-8 assay was performed to detect the proliferation of $\mathrm{HaCaT}$ cells. The results demonstrated that WT1 overexpression promoted the proliferation of $\mathrm{HaCaT}$ cells, particularly on days 4 and 5 following transfection (Fig. 3B). Apoptosis analysis was subsequently performed. The results of the flow cytometry revealed that the proportion 
A

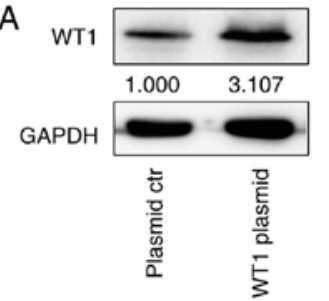



GAPDH $\quad 2777.0 \quad 3173.3$
B

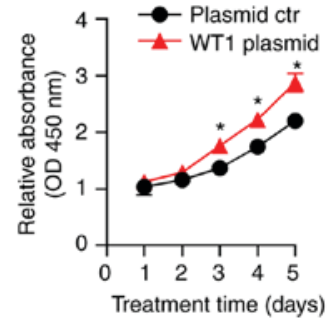

C

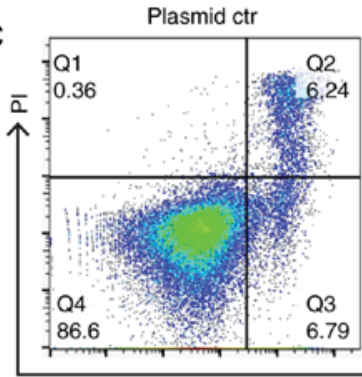

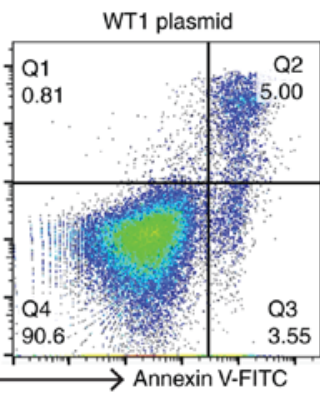
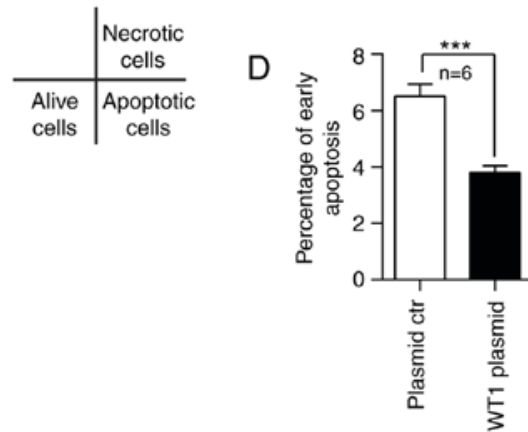

Figure 3. Overexpressing WT1 in keratinocytes promotes proliferation and inhibits apoptosis. (A) HaCaT cells were transfected with a WT1 overexpression or control plasmid for $48 \mathrm{~h}$, and the protein expression of WT1 was detected. (B) HaCaT cells were transfected with a WT1 overexpression or control plasmid, and the cells were collected on the indicated days to detect the proliferation status using a Cell Counting Kit-8 assay ( $\mathrm{n}=6$; $\mathrm{D} 3$, $\mathrm{P}=0.0216$; $\mathrm{D} 4$, $\mathrm{P}=0.0356$; $\mathrm{D} 5, \mathrm{P}=0.0188)$. (C) HaCaT cells were transfected with a WT1 overexpression or control plasmid, and the cells were harvested after $48 \mathrm{~h}$ for the apoptosis assay ( $\mathrm{n}=6)$. (D) Statistical analysis of the data is presented $(\mathrm{P}=0.0003)$. All experiments were repeated a minimum of three times. Data are presented as the mean \pm standard error of the mean. ${ }^{*} \mathrm{P}<0.05,{ }^{* * * *} \mathrm{P}<0.001$ vs. plasmid ctr. Two-tailed unpaired Student's t-tests (B and D) were used. WT1, Wilms' tumor 1 ; D, day; ctr, control; FITC, fluorescein isothiocyanate; PI, propidium iodide; OD, optical density.

of Annexin-positive and propidium iodide-negative cells, which represent early apoptotic cells, was significantly decreased in the WT1 plasmid transfection group compared with the control group (Fig. 3C and D).

Knockdown of WT1 in keratinocytes inhibits proliferation and promotes apoptosis. To further investigate the functional mechanism of WT1 in the pathogenesis of psoriasis, WT1 expression was knocked down in HaCaT cells using siRNA. The western blot analysis demonstrated that WT1 expression was significantly inhibited in the HaCaT cells transfected with WT1 siRNA compared with the negative control (Fig. 4A). The transfected $\mathrm{HaCaT}$ cells were harvested at different time points for the CCK- 8 assay. The results revealed that the proliferation ability of $\mathrm{HaCaT}$ cells was significantly decreased in the WT1 siRNA-transfected group compared with the control group (Fig. 4B). In addition, the effect of WT1 knockdown on the apoptosis of HaCaT cells was detected by flow cytometry, and it was observed that the proportion of early apoptotic HaCaT cells was clearly increased in the WT1 siRNA-transfected group compared with the control siRNA group (Fig. 4C and D).

WT1 expression is induced by proinflammatory factors. It has been previously demonstrated that epidermal keratinocytes are responsive to immune cell-derived cytokines, including IFNs, IL-17 and IL-22, which are increased in the skin lesions of psoriasis (27). To investigate the possible mechanisms associated with WT1 upregulation, HaCaT cells were stimulated with IL-17A, IFN- $\gamma$ and IL-22. The results of RT-qPCR demonstrated that cytokines at higher concentrations, including IFN- $\gamma$, IL-17A and IL-22, promoted WT1 mRNA expression (Fig. 5). These results indicated that the overexpression of WT1 may be due to the stimulation of proinflammatory factors in the local skin lesions of patients with psoriasis.

\section{Discussion}

Excessive proliferation and the abnormal apoptosis of keratinocytes serve an important role in the formation of psoriatic skin lesions (28). Numerous cellular growth and metabolism-associated genes have been reported to be dysregulated in psoriasis skin lesions. The WT1 gene, frequently mutated in numerous cancer types, encodes transcription factor WT1, which regulates apoptosis and cell cycle progression (29-31). In normal tissues, WT1 is an important regulator of cell growth and development (32). Accumulating evidence has demonstrated that WT1 has an oncogenic function in tumorigenesis. WT1 knockdown by a WT1 antisense oligomer or WT1 specific short hairpin RNA inhibits the growth of cancer cells expressing WT1 (33-35). Furthermore, overexpression of WT1 promotes cell growth, migration and invasion, and also inhibits cellular apoptosis under certain conditions (36). However, there are no reports about WT1 mutation in psoriasis as yet, to the best of our knowledge, and there is poor understanding as to whether the dysregulation of WT1 is involved in the abnormal growth of keratinocytes observed in psoriasis.

The present study demonstrated that the expression of WT1 was low in normal skin and significantly upregulated in non-lesional and lesional skins from patients with psoriasis and the IMQ-induced psoriasis-like mouse model, suggesting that the alteration in WT1 gene expression may be involved in the early pathogenesis of psoriasis. The expression of WT1 was also positively correlated with PASI scores. In addition, the 
A



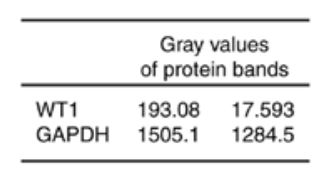



C

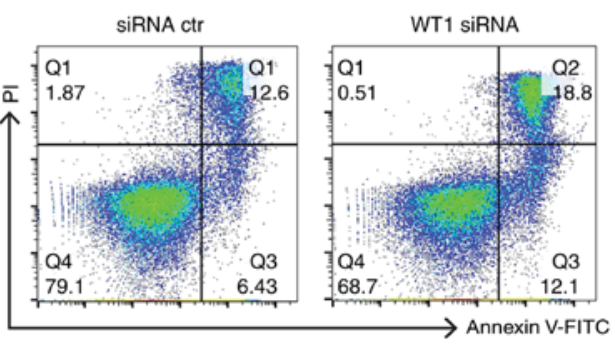



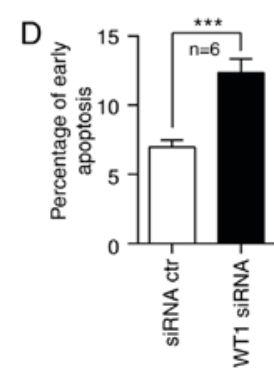

Figure 4. Knockdown of WT1 in keratinocytes inhibits proliferation and promotes apoptosis. (A) HaCaT cells were transfected with WT1 siRNA or control siRNA for $48 \mathrm{~h}$, and the protein expression of WT1 was detected. (B) HaCaT cells were transfected with WT1 siRNA or control siRNA, and the cells were collected on the indicated days to detect the proliferation status by Cell Counting Kit-8 assay ( $\mathrm{n}=6$; D4, P=0.0454; D5, P=0.0021). (C) HaCaT cells were transfected with WT1 or control siRNA, then the cells were harvested after $48 \mathrm{~h}$ for the apoptosis assay (n=6). (D) Statistical analysis of the data is presented $(\mathrm{P}=0.0007)$. All experiments were repeated a minimum of three times. Data are presented as the mean \pm standard error of the mean. ${ }^{*} \mathrm{P}<0.05,{ }^{* * *} \mathrm{P}<0.01$, ${ }^{* * *} \mathrm{P}<0.001$ vs. siRNA ctr. Two-tailed unpaired Student's t-tests (B and D) were used. WT1, Wilms' tumor 1; siRNA, small interfering RNA; D, day; ctr, control; FITC, fluorescein isothiocyanate; PI, propidium iodide; OD, optical density.

A

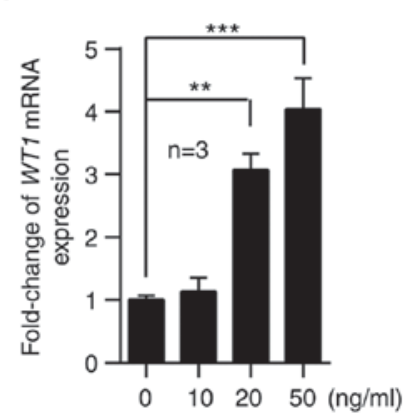

B

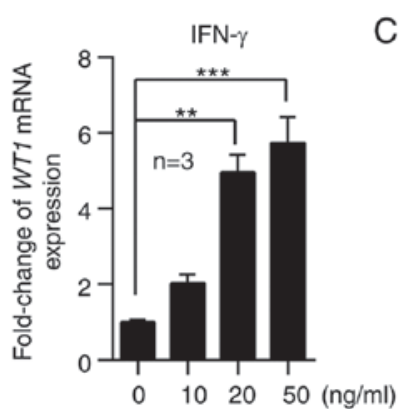

C

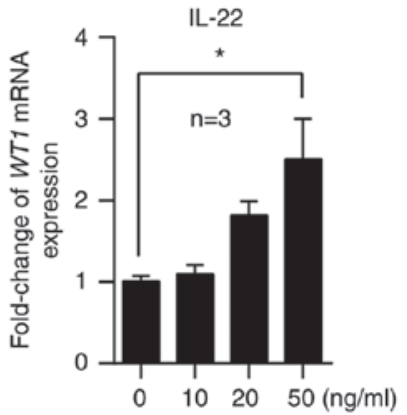

Figure 5. WT1 expression is induced by proinflammatory factors. HaCaT cells were stimulated with $0,10,20$ or $50 \mathrm{ng} / \mathrm{ml}$ (A) IL-17A, (B) IFN- $\gamma$ and (C) IL-22 for $24 \mathrm{~h}$. The cells were collected and analyzed to detect the mRNA expression of WT1 ( $\mathrm{n}=3)$. Compared with the control group, the mRNA expression of WT1 was increased in HaCaT cells stimulated with IL-17A (20 vs. 0 ng/ml, P=0.003; 50 vs. 0 ng/ml, P=0.0001), IFN- $\gamma(20$ vs. 0 ng/ml, P=0.001; 50 vs. 0 ng/ml, $\mathrm{P}=0.0001)$ and IL-22 (50 vs. $0 \mathrm{ng} / \mathrm{ml}, \mathrm{P}=0.011)$. All experiments were repeated a minimum of three times. The data are presented as the mean \pm standard error of the mean. ${ }^{*} \mathrm{P}<0.05,{ }^{* *} \mathrm{P}<0.01,{ }^{* * *} \mathrm{P}<0.001$. One-way analysis of variance with Dunnett's post hoc test was used. WT1, Wilms' tumor 1 ; IFN, interferon; IL, interleukin.

increased WT1 was limited to the epidermis of psoriatic skin, and it promoted the proliferation and inhibited the apoptosis of keratinocytes. WT1 is frequently involved in the regulation of cell growth and apoptosis through different target genes and signaling pathways, including C-myc (37), cellular tumor antigen p53-mediated cellular apoptosis (38), and the mitogen activated protein kinase and Janus kinase-signal transducer and activator of transcription signaling pathways (39). The majority of these target genes and signaling pathways are also involved in the pathogenesis of psoriasis (40-42). A previous study reported that WT1 mediates keratinocyte growth factor (KGF) signaling in breast cancer cells, which promotes DNA synthesis, cell proliferation and migration (43). KGF has been demonstrated to be upregulated in the upper dermis of psoriatic skin and its expression is correlated with keratinocyte growth (44). Therefore, it was speculated that WT1 promotes keratinocyte proliferation by regulating the downstream KGF signaling pathway in patients with psoriasis.

Although the initial events triggering a psoriatic lesion remain unclear, pro-inflammatory cytokines, including IL-17A, IL-22 and IFN- $\gamma$, may drive keratinocyte hyperproliferation and aberrant differentiation in psoriasis. A previous study suggested that the average protein expression levels of IL-17A, IFN- $\gamma$ and IL-22 in the cell culture supernatant of psoriatic $\mathrm{CD}^{+} \mathrm{T}$ cells are on the order of magnitude $\mathrm{ng} / \mathrm{ml}$ (45). In accordance with the literature (46), HaCaT cells were with $0,10,20$ or $50 \mathrm{ng} / \mathrm{ml} \mathrm{IL-17A,} \mathrm{IFN-} \gamma$ and IL-22 to examine their effect on the expression of WT1. It was demonstrated that these inflammatory cytokines were capable of inducing the overexpression of WT1 to varying degrees. It is widely 
accepted that the activation of nuclear factor (NF)- $\kappa \mathrm{B}$ transcription factors, which are crucial mediators involved in the pathogenesis of psoriasis (47), is a common downstream event following the stimulation of each of these cytokines (48-50). The present results provide a possible novel mechanism through which inflammatory cytokines may stimulate the upregulation of WT1 in psoriatic skin. This process may be mediated by $\mathrm{NF}-\kappa \mathrm{B}$ signaling, which serves an essential role in cell cycle regulation in the pathogenesis of psoriasis.

In conclusion, the results of the present study demonstrated that inflammatory cytokines induced the overexpression of WT1, which mediated the excessive proliferation and inhibited the apoptosis of keratinocytes in psoriasis. To the best of our knowledge, the present study is the first to focus on the expression and role of WT1 in psoriasis, and a potential novel factor associated with the pathogenesis of psoriasis has been revealed. However, further research is required to confirm these findings and investigate the possible molecular mechanisms of WT1 in psoriasis.

\section{Acknowledgements}

The authors thank Professor Zhang Janzhong (Department of Dermatology, Peking University People's Hospital) and Professor Zheng Min (Department of Dermatology, The Second Affiliated Hospital, Zhejiang University School of Medicine) for their advice on the experimental design.

\section{Funding}

The present study was supported by: The National Science Fund for Excellent Young Scholars (grant no. 81522038) and the Project of Innovation-driven Plan of Central South University (grant no. 2016CX029) to MZ; the National Natural Science Foundation of China (grant no. 81573051) to YS; the Key Program of National Natural Science Foundation of China (grant no. 81430074) to QL; the Fundamental Research Funds for the Central Universities of Central South University (grant no. 2016zzts143) to RW; and the National Natural Science Foundation of China (grant no. 81502732) to YL.

\section{Availability of data and materials}

All data generated or analyzed during this study are included in this published article.

\section{Authors' contributions}

RW performed most of the experiments, analyzed the data and wrote the manuscript. YLiao performed the cell transfection. WS, YLiu and YS collected the clinical samples, evaluated the PASI score of patients and analyzed the correlation between WT1 expression and PASI score. JZ, MZhe and GC critically revised the manuscript, and provided technical support and suggestions. QL and MZha designed and supervised the study.

\section{Ethics approval and consent to participate}

The present study was approved by the Ethics Committee of the Second Xiangya Hospital of Central South University
(Changsha, China). Written informed consent was obtained from all subjects. All procedures involving animals were approved and supervised by the Animal Care and Use Committee of the Second Xiangya Medical School of Central South University.

\section{Patient consent for publication}

Not applicable.

\section{Competing interests}

The authors declare that they have no competing interests.

\section{References}

1. Schön MP and Boehncke WH: Psoriasis. N Engl J Med 352: 1899-1912, 2005.

2. Lowes MA, Bowcock AM and Krueger JG: Pathogenesis and therapy of psoriasis. Nature 445: 866-873, 2007.

3. Boehncke WH and Schön MP: Psoriasis. Lancet 386: 983-994, 2015.

4. Suárez-Fariñas M, Li K, Fuentes-Duculan J, Hayden K, Brodmerkel C and Krueger JG: Expanding the psoriasis disease profile: Interrogation of the skin and serum of patients with moderate-to-severe psoriasis. J Invest Dermatol 132: 2552-2564, 2012.

5. Tan NS, Michalik L, Noy N, Yasmin R, Pacot C, Heim M, Flühmann B, Desvergne B and Wahli W: Critical roles of PPAR beta/delta in keratinocyte response to inflammation. Genes Dev 15: 3263-3277, 2001.

6. Croxford AL, Karbach S, Kurschus FC, Wörtge S, Nikolaev A, Yogev N, Klebow S, Schüler R, Reissig S, Piotrowski C, et al: IL-6 regulates neutrophil microabscess formation in IL-17A-driven psoriasiform lesions. J Invest Dermatol 134: 728-735, 2014.

7. Uyemura K, Yamamura M, Fivenson DF, Modlin RL and Nickoloff BJ: The cytokine network in lesional and lesion-free psoriatic skin is characterized by a T-helper type 1 cell-mediated response. J Invest Dermatol 101: 701-705, 1993.

8. Zaba LC, Suárez-Fariñas M, Fuentes-Duculan J, Nograles KE, Guttman-Yassky E, Cardinale I, Lowes MA and Krueger JG: Effective treatment of psoriasis with etanercept is linked to suppression of IL-17 signaling, not immediate response TNF genes. J Allergy Clin Immunol 124: 1022-1110.e1-e395, 2009.

9. Schön M, Behmenburg C, Denzer D and Schön MP: Pathogenic function of IL-1 beta in psoriasiform skin lesions of flaky skin (fsn/fsn) mice. Clin Exp Immunol 123: 505-510, 2001.

10. Gisondi P, Gubinelli E, Cocuroccia B and Girolomoni G: Targeting tumor necrosis factor-alpha in the therapy of psoriasis. Curr Drug Targets Inflamm Allergy 3: 175-183, 2004.

11. Green LM, Wagner KJ, Campbell HA, Addison K and Roberts SG: Dynamic interaction between WT1 and BASP1 in transcriptional regulation during differentiation. Nucleic Acids Res 37: 431-440, 2009.

12. Park S, Tomlinson G, Nisen P and Haber DA: Altered trans-activational properties of a mutated WT1 gene product in a WAGR-associated Wilms' tumor. Cancer Res 53: 4757-4760, 1993.

13. Bruening W, Gros P, Sato T, Stanimir J, Nakamura Y, Housman D and Pelletier J: Analysis of the 11p13 Wilms' tumor suppressor gene (WT1) in ovarian tumors. Cancer Invest 11: 393-399, 1993.

14. Silberstein GB, Van Horn K, Strickland P, Roberts CT Jr and Daniel CW: Altered expression of the WT1 wilms tumor suppressor gene in human breast cancer. Proc Natl Acad Sci USA 94: 8132-8137, 1997.

15. Oji Y, Yano M, Nakano Y, Abeno S, Nakatsuka S, Ikeba A, Yasuda T, Fujiwara Y, Takiguchi S, Yamamoto $\mathrm{H}$, et al: Overexpression of the Wilms' tumor gene WT1 in esophageal cancer. Anticancer Res 24: 3103-3108, 2004.

16. Keilholz U, Menssen HD, Gaiger A, Menke A, Oji Y, Oka Y, Scheibenbogen C, Stauss H, Thiel E and Sugiyama H: Wilms tumour gene 1 (WT1) in human neoplasia. Leukemia 19: 1318-1323, 2005 
17. Oji Y, Nakamori S, Fujikawa M, Nakatsuka S, Yokota A, Tatsumi N, Abeno S, Ikeba A, Takashima S, Tsujie M, et al: Overexpression of the Wilms' tumor gene WT1 in pancreatic ductal adenocarcinoma. Cancer Sci 95: 583-587, 2004.

18. Gao SM, Yang JJ, Chen CQ, Chen JJ, Ye LP, Wang LY, Wu JB, $\mathrm{Xing} \mathrm{CY}$ and $\mathrm{Yu} \mathrm{K}$ : Pure curcumin decreases the expression of WT1 by upregulation of miR-15a and miR-16-1 in leukemic cells. J Exp Clin Cancer Res 31: 27, 2012.

19. Loeb DM and Sukumar S: The role of WT1 in oncogenesis: Tumor suppressor or oncogene? Int J Hematol 76: 117-126, 2002

20. Hohenstein P and Hastie ND: The many facets of the Wilms tumour gene, WT1. Hum Mol Genet 15 Spec No 2: R196-R201, 2006.

21. Huff V: Wilms' tumours: About tumour suppressor genes, an oncogene and a chameleon gene. Nat Rev Cancer 11: 111-121, 2011

22. Armstrong AW, Parsi K, Schupp CW, Mease PJ and Duffin KC Standardizing training for psoriasis measures: Effectiveness of an online training video on Psoriasis Area and Severity Index assessment by physician and patient raters. JAMA Dermatol 149: $577-582,2013$

23. Malkic Salihbegovic E, Hadzigrahic N and Cickusic AJ: Psoriasis and metabolic syndrome. Med Arch 69: 85-87, 2015.

24. van der Fits L, Mourits S, Voerman JS, Kant M, Boon L, Laman JD, Cornelissen F, Mus AM, Florencia E, Prens EP and Lubberts E: Imiquimod-induced psoriasis-like skin inflammation in mice is mediated via the IL-23/IL-17 axis. J Immunol 182 5836-5845, 2009.

25. Livak KJ and Schmittgen TD: Analysis of relative gene expression data using real-time quantitative PCR and the 2(-Delta Delta C(T)) method. Methods 25: 402-408, 2001

26. Mak RK, Hundhausen C and Nestle FO: Progress in understanding the immunopathogenesis of psoriasis. Actas Dermosifiliogr 100 (Suppl 2): S2-S13, 2009.

27. Nestle FO, Kaplan DH and Barker J: Psoriasis. N Engl J Med 361: 496-509, 2009

28. Ragaz A and Ackerman AB: Evolution, maturation, and regression of lesions of psoriasis. New observations and correlation of clinical and histologic findings. Am J Dermatopathol 1: 199-214, 1979.

29. Scharnhorst V, Dekker P, van der Eb AJ and Jochemsen AG: Internal translation initiation generates novel WT1 protein isoforms with distinct biological properties. J Biol Chem 274: 23456-23462, 1999

30. Yang L, Han Y, Suarez Saiz F and Minden MD: A tumor suppressor and oncogene: The WT1 story. Leukemia 21: 868-876, 2007.

31. Scharnhorst V, van der Eb AJ and Jochemsen AG: WT1 proteins: Functions in growth and differentiation. Gene 273: 141-161, 2001.

32. Wagner KD, Cherfils-Vicini J, Hosen N, Hohenstein P, Gilson E, Hastie ND, Michiels JF and Wagner N: The Wilms' tumour suppressor Wt1 is a major regulator of tumour angiogenesis and progression. Nat Commun 5: 5852, 2014.

33. Algar EM, Khromykh T, Smith SI, Blackburn DM, Bryson GJ and Smith PJ: A WT1 antisense oligonucleotide inhibits proliferation and induces apoptosis in myeloid leukaemia cell lines. Oncogene 12: 1005-1014, 1996.

34. Yamagami T, Sugiyama $H$, Inoue $K$, Ogawa $H$, Tatekawa $T$, Hirata M, Kudoh T, Akiyama T, Murakami A and Maekawa T: Growth inhibition of human leukemic cells by WT1 (Wilms tumor gene) antisense oligodeoxynucleotides: Implications for the involvement of WT1 in leukemogenesis. Blood 87: 2878-2884, 1996.
35. Tatsumi N, Oji Y, Tsuji N, Tsuda A, Higashio M, Aoyagi S, Fukuda I, Ito K, Nakamura J, Takashima S, et al: Wilms' tumor gene WT1-shRNA as a potent apoptosis-inducing agent for solid tumors. Int J Oncol 32: 701-711, 2008

36. Xu C, Wu C, Xia Y, Zhong Z, Liu X, Xu J, Cui F, Chen B, Røe OD, Li A and Chen Y: WT1 promotes cell proliferation in non-small cell lung cancer cell lines through up-regulating cyclin D1 and p-pRb in vitro and in vivo. PLoS One 8: e68837, 2013.

37. Hewitt SM, Hamada S, McDonnell TJ, Rauscher FJ III and Saunders GF: Regulation of the proto-oncogenes bcl-2 and c-myc by the Wilms' tumor suppressor gene WT1. Cancer Res 55: 5386-5389, 1995.

38. Maheswaran S, Englert C, Bennett P, Heinrich G and Haber DA: The WT1 gene product stabilizes p53 and inhibits p53-mediated apoptosis. Genes Dev 9: 2143-2156, 1995.

39. Li X, Li Y, Yuan T, Zhang Q, Jia Y, Li Q, Huai L, Yu P, Tian Z, Tang K, et al: Exogenous expression of WT1 gene influences U937 cell biological behaviors and activates MAPK and JAK-STAT signaling pathways. Leuk Res 38: 931-939, 2014.

40. Kim BH,Lee JM,Jung YG, Kim S and Kim TY: Phytosphingosine derivatives ameliorate skin inflammation by inhibiting NF- $\kappa \mathrm{B}$ and JAK/STAT signaling in keratinocytes and mice. J Invest Dermatol 134: 1023-1032, 2014.

41. Moorchung N, Vasudevan B, Dinesh Kumar S and Muralidhar A: Expression of apoptosis regulating proteins p53 and bcl-2 in psoriasis. Indian J Pathol Microbiol 58: 423-426, 2015.

42. Casado M, Martin M, Muñoz A and Bernal J: Vitamin D3 inhibits proliferation and increases c-myc expression in fibroblasts from psoriatic patients. J Endocrinol Invest 21: 520-525, 1998.

43. Zang XP, Pento JT and Tari AM: Wilms' tumor 1 protein and focal adhesion kinase mediate keratinocyte growth factor signaling in breast cancer cells. Anticancer Res 28: 133-137, 2008.

44. Kovacs D, Falchi M, Cardinali G, Raffa S, Carducci M, Cota C, Amantea A, Torrisi MR and Picardo M: Immunohistochemical analysis of keratinocyte growth factor and fibroblast growth factor 10 expression in psoriasis. Exp Dermatol 14: 130-137, 2005.

45. Wu R, Zeng J, Yuan J, Deng X, Huang Y, Chen L, Zhang P, Feng H, Liu Z, Wang Z, et al: MicroRNA-210 overexpression promotes psoriasis-like inflammation by inducing Th1 and Th17 cell differentiation. J Clin Invest 128: 2551-2568, 2018.

46. Yan S, Xu Z, Lou F, Zhang L, Ke F, Bai J, Liu Z, Liu J, Wang H, Zhu $\mathrm{H}$, et al: NF- $\kappa \mathrm{B}$-induced microRNA-31 promotes epidermal hyperplasia by repressing protein phosphatase 6 in psoriasis. Nat Commun 6: 7652,2015

47. Goldminz AM, Au SC, Kim N, Gottlieb AB and Lizzul PF: NF- $\kappa$ B: An essential transcription factor in psoriasis. J Dermatol Sci 69: 89-94, 2013.

48. Hang do TT, Song JY, Kim MY, Park JW and Shin YK: Involvement of NF-KB in changes of IFN- $\gamma$-induced CIITA/MHC-II and iNOS expression by influenza virus in macrophages. Mol Immunol 48: 1253-1262, 2011.

49. Wu Y, Zhu L, Liu L, Zhang J and Peng B: Interleukin-17A stimulates migration of periodontal ligament fibroblasts via p38 MAPK/NF- $\kappa$ B-dependent MMP-1 expression. J Cell Physiol 229: 292-299, 2014.

50. Gelebart P, Zak Z, Dien-Bard J, Anand M and Lai R: Interleukin 22 signaling promotes cell growth in mantle cell lymphoma. Transl Oncol 4: 9-19, 2011. 